\title{
CHANNELS
}

\section{Dual eicosanoid activity}

Immunol. 191, 6136-6146 (2013)

Macrophage cells are central players in the immune system and can be activated by different stimuli, resulting in two different downstream pathways. The innate activation pathway results from stimulation by pathogen lipopolysaccharide (LPS), whereas the second alternative pathway is activated by various cytokines and glucocorticoids. Voltage-dependent potassium channels $\left(\mathrm{K}_{\mathrm{v}}\right)$ are regulated by both pathways but in opposite directions: the innate pathway triggers $\mathrm{K}_{\mathrm{v}}$ outward current, whereas alternative activation downregulates $\mathrm{K}_{\mathrm{v}}$ current. Also, innate activation increases, whereas alternative activation decreases, the ratio of the $\mathrm{K}_{\mathrm{v}} 1.3$ to $\mathrm{K}_{\mathrm{v}} 1.5$ isoforms in these cells. To explore the connections between the two types of activation and the role of potassium channels, Moreno et al. monitored the responses in bone marrow-derived macrophages to the endogenous anti-inflammatory eicosanoids 15-epi-LXA $\left(\mathrm{e}-\mathrm{LXA}_{4}\right)$ and the less potent lipoxin $\mathrm{A}_{4}\left(\mathrm{LXA}_{4}\right)$, which have been shown to inhibit the alternative activation pathway via their receptor ALX. e-LXA $\mathrm{A}_{4}$ acted on $\mathrm{K}_{\mathrm{v}}$ channels to generate inactivating currents and led to inhibition of the pro-inflammatory transcription factor NF- $\kappa$ B via modulation of its activator IKK $\beta$. e-LXA $\mathrm{LA}_{4}$ also prevented changes in $\mathrm{K}_{\mathrm{v}} 1.3 / \mathrm{K}_{\mathrm{v}} 1.5$ stoichiometry through changes in the innate LPS signaling pathway that specifically involve ALX receptors that then modulate $\mathrm{K}_{\mathrm{v}} 1.3$ channels. These results

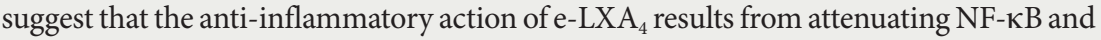
additionally by modulating potassium currents in macrophages.

\section{RNA STRUCTURE}

\section{Folding in the wild}

Nature doi: $10.1038 /$ nature12756

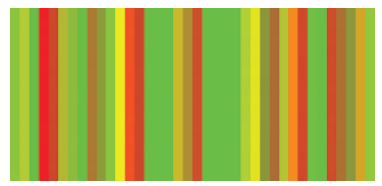

Chemical probing methods-in which folded RNAs are modified by chemoselective reagents and analyzed by reverse transcription-are standard approaches for in vitro RNA secondary structure determination. However, reagents such as dimethyl sulfate (DMS), which methylates adenosine and cytosine bases in unpaired RNA regions, have seen limited application in cells and organisms. Ding et al. now report a method for genome-wide RNA secondary structure analysis in vivo. Using 'structure-seq', which combines in vivo DMS modification with deep sequencing, the authors profiled Arabidopsis thaliana seedlings and obtained single-nucleotide resolution modification data on over 10,000 transcripts. Analysis of the DMS modification profiles of the mRNA transcriptome revealed cases where RNA secondary structure may have a direct role in regulating gene expression. For instance, enhanced DMS modification profiles upstream of start codons correlate with high ribosome association and translational efficiency. In contrast, mRNA regions upstream of alternative polyadenylation or alternative splice sites showed lower DMS modification frequency. Interestingly, mRNAs coding for stress response proteins displayed evidence for greater structural flexibility than those involved in housekeeping roles, a property that may be functionally important for environmentally responsive RNAs. In addition to providing an approach for probing RNA structure under physiological conditions, structure-seq also highlights practical gaps between in vitro, in vivo and in silico RNA structural prediction methods that merit the community's attention.

TLS

\section{TRANSLATION}

\section{Makes sense}

Angew. Chem. Int. Ed. Engl.

doi:10.1002/anie.201308584

Translational recoding to incorporate non-natural or otherwise modified amino acids has generally focused on stop codons as the modifiable triplet. However, recent studies have shown that incorporation of selenocysteine $(\mathrm{Sec})$ is possible at low levels at the sense codons UUA (leucine) and UGG (tryptophan). To investigate whether these low levels were due to competition with the 20 canonical amino acids generally or whether other codons might be more amenable to replacement, Bröcker et al. systematically tested each of the 64 codons to see whether Sec could be inserted and to what extent. Both formate dehydrogenase and thioredoxin reductase naturally include and are functionally dependent on Sec, meaning that activity assays provide a straightforward and quantitative proxy for Sec incorporation. Surprisingly, in formate dehydrogenase, 58 codons could be translated with Sec, yielding activities ranging from $12 \%$ to $100 \%$ of the parent sequence. The fully active codon replacements included all three stop codons as well as 15 sense codons for which the proteins not only were active but also were expressed at much higher levels than those using stop codons. The most active of these, UAC, showed the same behavior in recoding thioredoxin reductase, yielding wild-type activity and better yields than the most commonly used stop codon, UGA. These data raise new questions about translational fidelity and create new opportunities for protein engineering.

TARGET IDENTIFICATION

\section{Chemical landing pad}

Nat. Biotechnol. doi:10.1038/nbt.2776

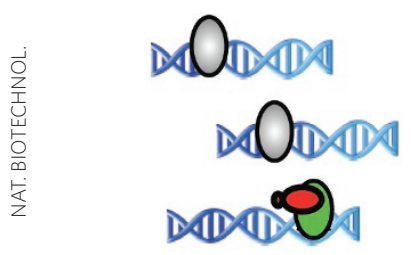

Certain classes of small molecules can elicit their effects on cellular function through alterations of either chromatinbinding proteins or on chromatin itself. Although techniques such as chromatin immunoprecipitation (ChIP)-seq have been useful to globally map the genomic location of transcription factor binding, there has been a growing need to extend these approaches to small molecules. Anders et al. now report on a new assay called Chem-seq, which uses the same techniques as ChIP but relies on a biotinylated small molecule to allow enrichment using streptavidin beads. This approach can either be used directly on living cells or applied to a cellular extract in cases where the probe cannot penetrate the cell. The authors performed Chem-seq on a biotinylated form of JQ1, an inhibitor of the BET bromodomain family. Comparison of the JQ1 binding sites with ChIP analysis of the individual BET family members $\mathrm{BRD} 2, \mathrm{BRD} 3$ and BRD4 revealed a strong colocalization in DNA binding sites to BRD4, in particular. They tested their in vitro approach on AT5719, an inhibitor of the cyclin-dependent kinase CDK9, and observed a strong correlation in chromatinbinding sites between AT5719 and CDK9. Taken together, Chem-seq could be a useful tool to assist investigators to better elucidate the mechanism of action of small-molecule compounds.

Written by Mirella Bucci, Amy Donner, Catherine Goodman, Grant Miura and Terry L. Sheppard 